\title{
Downstaging and resection of hepatocellular carcinoma in patients with extrahepatic metastases after stereotactic therapy
}

\author{
Xiaobo Yang", Haifeng Xu", Bangyou Zuo", Xu Yang", Jin Bian, Junyu Long, Dongxu Wang, \\ Junwei Zhang, Cong Ning, Yanyu Wang, Ziyu Xun, Yunchao Wang, Xin Lu, Yilei Mao, Xinting Sang, \\ Haitao Zhao
}

Department of Liver Surgery, State Key Laboratory of Complex Severe and Rare Diseases, Peking Union Medical College Hospital, Chinese Academy of Medical Sciences and Peking Union Medical College (CAMS \& PUMC), Beijing, China

Contributions: (I) Conception and design: H Zhao, X Yang; (II) Administrative support: H Zhao, X Sang; (III) Provision of study materials or patients: $\mathrm{H} \mathrm{Xu}$, B Zuo, X Yang; (IV) Collection and assembly of data: B Zuo, X Yang; (V) Data analysis and interpretation: All authors; (VI) Manuscript writing: All authors; (VII) Final approval of manuscript: All authors.

"These authors contributed equally to this work.

Correspondence to: Haitao Zhao; Xinting Sang; Yilei Mao; Xin Lu. Department of Liver Surgery, State Key Laboratory of Complex Severe and Rare Diseases, Peking Union Medical College Hospital, Chinese Academy of Medical Sciences and Peking Union Medical College (CAMS \& PUMC), Beijing, China. Email: ZhaoHT@pumch.cn; sangxt@pumch.cn; yileimao@126.com; luxinln@163.com.

Background: A combination of tyrosine kinase inhibitors (TKIs) and anti-PD-1 antibodies with local regional therapy has elicited yield substantial clinical benefits in patients who have hepatocellular carcinoma (HCC) with extrahepatic metastases. Using this treatment strategy to convert HCC patients with extrahepatic metastases from unresectable to resectable has not yet been reported.

Methods: Consecutive hepatocellular carcinoma patients with extrahepatic metastases who received firstline therapy with a combination of TKIs and anti-PD-1 antibodies and at least one local regional therapy were analysed.

Results: Nine patients with localized disease who received first-line systemic therapy were enrolled. At baseline, all of them had oligometastatic disease, namely, Barcelona Clinic Liver Cancer stage C (or Chinese Liver Cancer stage IIIB). The most common treatment administered was lenvatinib plus anti-PD-1 antibody and transarterial chemoembolization, and the median time span from systemic therapy to surgery was 3.2 (IQR, 2.8-6.2) months. Three patients achieved a pathological complete response. Six patients underwent laparoscopic surgery, and the other 3 patients underwent open surgery. After a median follow-up of 10.2 (IQR, 8.6-20.0) months, 7 patients survived without disease recurrence, and 2 experienced tumour recurrence. All patients had any-grade AEs, and $55.6 \%$ of the patients experienced grade 3 AEs. Fatigue was the most common AE, followed by elevated aminotransferase levels and hypertension.

Conclusions: Stereotactic therapy is a feasible conversion therapy for HCC patients with extrahepatic metastases to become resectable. This is the first study to analyse therapeutic outcomes of patients receiving these therapies for HCC with extrahepatic metastases.

Keywords: Hepatocellular carcinoma (HCC); stereotactic therapy; PD-1; systemic therapy; conversion surgery

Submitted May 12, 2021. Accepted for publication Jun 18, 2021.

doi: $10.21037 / \mathrm{hbsn}-21-188$

View this article at: https://dx.doi.org/10.21037/hbsn-21-188 


\section{Introduction}

Hepatocellular carcinoma (HCC) is the sixth most common cancer worldwide and the second most common cause of cancer-related death in China $(1,2)$. Most patients with HCC are diagnosed at an advanced stage; thus, these patients are not suitable for local radical surgery, and effective treatments are lacking $(3,4)$. Sorafenib is an oral multi-kinase inhibitor that was found to improve overall survival (OS) in the SHARP and Asia-Pacific trials $(5,6)$. Later, lenvatinib and atezolizumab plus bevacizumab were found to be effective against advanced hepatocellular carcinoma, and together with sorafenib, they have all become first-line treatment strategies $(7,8)$. However, in real-world practice, due to the poor efficacy of a single drug, patients tend to receive immunotherapy and targeted combination therapy (9). We define this combination plus local regional treatment as stereotactic therapy, which we feel could truly reflect the treatment conditions of patients in the real world.

Conversion surgery, or the notion that surgical treatment aimed at R0 resection can be performed following stereotactic therapy for tumours initially considered unresectable due to technical and/or oncological reasons, is one of the new therapeutic approaches for unresectable cancer, and successful treatment outcomes have been reported, which is different from neoadjuvant therapy. Sato et al. described a combination of lenvatinib and TACE to convert patients with unresectable large hepatocellular carcinoma, with successful resections performed in their patient population (10). Takeda et al. revealed that regorafenib can be used to transform cases of unresectable hepatocellular carcinoma with vascular tumour thrombosis into resectable cases (11). Schwacha-Eipper et al. reported that immunotherapy can be used for conversion of unresectable patients with large hepatocellular carcinoma to liver transplantation (12).

In our report, we focused on real-world hepatocellular carcinoma patients with extrahepatic metastasis [Barcelona Clinic Liver Cancer (BCLC) stage C or Chinese Liver Cancer Stage (CNLC) IIIB] who received stereotactic therapy and were later converted to surgical resection. We preliminarily estimated the therapeutic efficacy and safety of stereotactic therapy. We present the following article in accordance with the STROBE reporting checklist (available at https://hbsn. amegroups.com/article/view/10.21037/hbsn-21-188/rc).

\section{Methods}

\section{Study population}

From May 2019 to March 2021, 9 HCC patients with extrahepatic metastasis who received stereotactic therapy at Peking Union Medical College Hospital (PUMCH) were enrolled in this study. HCC was diagnosed by multiphase computed tomography (CT) or magnetic resonance imaging (MRI) or fine needle aspiration pathology according to the guidelines of HCC (13). The demographics, laboratory results, surgical, pathological, stereotactic therapy regimens, safety assessment and grading, and clinical outcomes were recorded. The study is registered at ClinicalTrials.gov (identifier: NCT03892577). The study was conducted in accordance with the Declaration of Helsinki (as revised in 2013). And the study was approved by the Institutional Review Board (IRB) and Ethics Committee (EC) of Peking Union Medical College Hospital (PUMCH) (approval number: JS-1391) and informed consent was taken from all individual participants.

\section{Treatment and dosing}

Information regarding the dates of initiation and completion of treatment, initial dose, dose modifications, radiological evaluation, laboratory data, surgery data, and adverse events (AEs) during treatment were systematically collected. Lenvatinib was administered at a dosage of $12 \mathrm{mg}$ (for patients with a body weight $\geq 60 \mathrm{~kg}$ ) or $8 \mathrm{mg}$ (for patients with a body weight $<60 \mathrm{~kg}$ ) orally once a day. The PD-1 dose included a fixed dosage of $200 \mathrm{mg}$ (240 mg for toripalimab) every 3 weeks or a fixed dosage of $3 \mathrm{mg} / \mathrm{kg}$ body weight every 3 weeks.

\section{Outcome assessment}

The clinical objective response was measured by the mRECIST criteria (14) and evaluated by professional radiologists at the centre (PUMCH) who were blinded to therapeutic outcomes and clinicopathological features. Objective response rate (ORR) and disease control rate (DCR) were used to assess the efficacy of treatments.

Safety assessment and grading were recorded in the electronic medical records or collected by the investigators using the Common Terminology Criteria for Adverse Events (version 4.0) as a reference. 


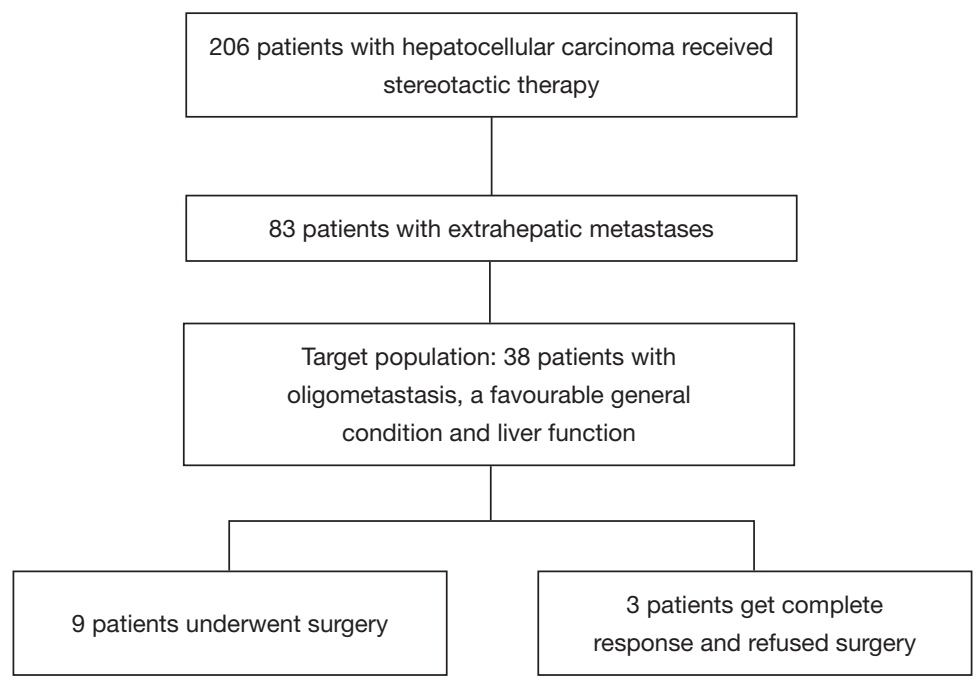

Figure 1 Patients flowchart.

\section{Statistical analysis}

The data from the cut-off date of March 31, 2021 were used to describe the baseline characteristics, efficacies, and adverse events. In order to compare the various variables, the $\chi^{2}$ test was performed appropriately and two-tailed $\mathrm{P}$ value $<0.05$ is considered significant. Statistical analyses were performed by SPSS (version 25).

\section{Results}

\section{Baseline characteristics of the study population}

A total of 206 hepatocellular carcinoma patients received stereotactic therapy, of which 83 patients had extrahepatic metastases. Among the 83 cases, 38 cases were our target population (oligometastasis, a favourable general condition and liver function). In the end, 3 patients got complete response and refused surgery and $9(9 / 38,23.7 \%)$ patients downstage the tumors and underwent conventional surgery. (Figure 1) The demographics and baseline characteristics of all 9 patients are summarized in Table 1. At the time of initial treatment, the median age was 52.0 (IQR, 40.5-57.5) years, and $22.2 \%$ of the total patients were female. All patients enrolled in the study had an ECOG score of zero and Child-Pugh stage A. Eight (88.9\%) patients had an aetiology of HBV or HBV plus alcohol abuse, and six $(66.7 \%)$ patients had a habit of smoking. The diameter of the major tumour was 8.0 (IQR, 6.1-10.5) centimeters, and most of the patients had 1-2 tumours in the liver $(5 / 9,55.6 \%)$, and $3(33.3 \%)$ patients had portal vein invasion. All the nine patients had oligometastasis, which means a metastatic disease state with limited number and distribution, and usually refers to less than 5 metastases, and the primary lesion is stable as well (15). At baseline before stereotactic treatment, the most common site of metastasis was the abdominal cavity $(5 / 9,55.6 \%)$.

\section{Treatment and efficacy}

Most patients (7/9, 77.8\%) received lenvatinib plus PD-1, and $7(77.8 \%)$ patients received at least one locoregional treatment of transarterial chemoembolization (TACE) or radiotherapy. One patient received apatinib plus camrelizumab, and one patient received lenvatinib alone; the brain metastases in this patient disappeared, so they were down-staged and then underwent conversion surgery. The median time span from systemic therapy to surgery was 3.2 (IQR, 2.8-6.2) months. The stereotactic therapeutic regimens are shown in Figure 2.

Stereotactic treatment regimens administered in the process are shown in Table 2. Lesions in the liver and metastatic sites were observed in all cases at baseline and before surgery; the details are shown in Figure 3.

For all participants in our cohort, the median duration of follow-up was 10.2 (IQR, 8.6-19.9) months. All patients underwent complete radiological evaluations. Overall, all 9 patients $(100 \%)$ exhibited a decrease in tumour size from baseline (Figure 4). Among them, 5 patients achieved an objective response, all of whom showed a PR; no CR was observed. In the population with available radiological 
Table 1 Baseline characteristics of the study cohort

\begin{tabular}{|c|c|}
\hline Parameters & Total $(\mathrm{N}=9)$ \\
\hline Age, years (median, IQR) & $52.0(40.5-57.5)$ \\
\hline \multicolumn{2}{|l|}{ Sex, n (\%) } \\
\hline Female & $2(22.2)$ \\
\hline Male & $7(77.8)$ \\
\hline \multicolumn{2}{|l|}{ ECOG performance, n (\%) } \\
\hline 0 & $9(100.0)$ \\
\hline \multicolumn{2}{|l|}{ Aetiology, n (\%) } \\
\hline HBV & $4(44.4)$ \\
\hline HBV and alcohol & $4(44.4)$ \\
\hline NA & $1(11.1)$ \\
\hline Habit of smoking, n (\%) & $6(66.7)$ \\
\hline \multicolumn{2}{|l|}{ Child-Pugh stage, n (\%) } \\
\hline A5 & $9(100.0)$ \\
\hline \multicolumn{2}{|l|}{ CNLC stage, n (\%) } \\
\hline IIIB & $9(100.0)$ \\
\hline \multicolumn{2}{|l|}{ BCLC stage, n (\%) } \\
\hline C & $9(100.0)$ \\
\hline $\begin{array}{l}\text { Diameter of major tumour, cm } \\
\text { (median, IQR) }\end{array}$ & $8.0(6.1-10.5)$ \\
\hline \multicolumn{2}{|l|}{ Number of tumours in liver, $\mathrm{n}(\%)$} \\
\hline $1-2$ & $5(55.6)$ \\
\hline$\geq 3$ & $2(22.2)$ \\
\hline 0 & $2(22.2)$ \\
\hline \multicolumn{2}{|l|}{ Site of metastases, n (\%) } \\
\hline Lymph node & $1(11.1)$ \\
\hline Lung & $2(22.2)$ \\
\hline Brain & $1(11.1)$ \\
\hline Abdominal cavity & $5(55.6)$ \\
\hline Portal vein invasion, n (\%) & $3(33.3)$ \\
\hline
\end{tabular}

ECOG, Eastern Cooperative Oncology Group; HBV, hepatitis type B virus; CNLC, Chinese Liver Cancer Stage; BCLC, Barcelona Clinic Liver Cancer.

assessments, the best response in 4 (44.4\%) patients was SD. Therefore, the overall radiologically confirmed ORR was $55.6 \%$, and the DCR was $100 \%$.

\section{Surgery and perioperative conditions}

The median interval between the initiation of systemic

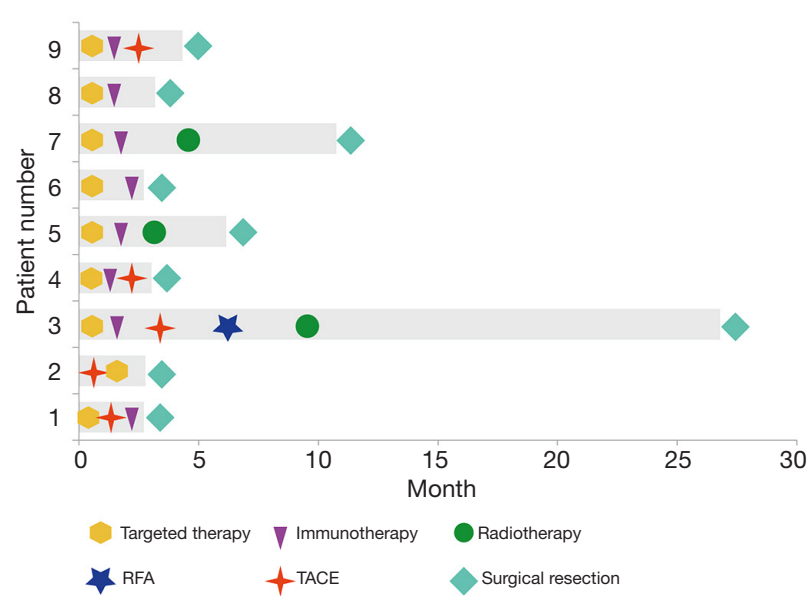

Figure 2 Overview of the stereotactic treatment regimens, and the $\mathrm{x}$-axis represented the treatment time, and the $\mathrm{y}$-axis represented different patients. Different symbols represented different therapy strategy.

therapy and surgery was 3.2 (IQR, 2.8-6.2) months. Six of the nine patients underwent laparoscopic surgery, and the other 3 patients underwent open surgery. The median time of surgery was 200 (IQR, 190-220) minutes, and the median volume of blood loss was 200 (IQR, 100-220) milliliters. The median postoperative hospital stay days was 6 (IQR, 5-8) days. Two patients experienced postoperative complications, namely, grade I (bile leakage) and grade IIIa (abdominal effusion requiring additional puncture) complications. A total of 3 patients $(33.3 \%)$ showed a pathological complete response (pCR), and no MVI was observed (Table 3).

\section{Safety}

In total, AEs during stereotactic therapy were reported in all 9 patients (100\%), but no grade 4-5 AEs occurred. In terms of severe AEs (SAEs), 5 (55.6\%) patients had grade 3 AEs (Table 4), which was similar to literature report $(\mathrm{P}=0.715)(16)$. The most common AEs (any grade) were fatigue (66.7\%), elevated aminotransferase (ALT or AST) levels (66.7\%) and hypertension (55.6\%). Most AEs that occurred during combination immunotherapy were safe, well tolerated and controlled. The most common grade $3 \mathrm{SAE}$ was hypertension $(22.2 \%)$, and after careful monitoring and treatment, blood pressure returned to normal. One patient had grade 3 asthma, but we did not have enough evidence to determine whether the $\mathrm{AE}$ was induced by stereotactic therapy because he had a history of asthma. 
Table 2 Characteristics of the stereotactic treatments administered

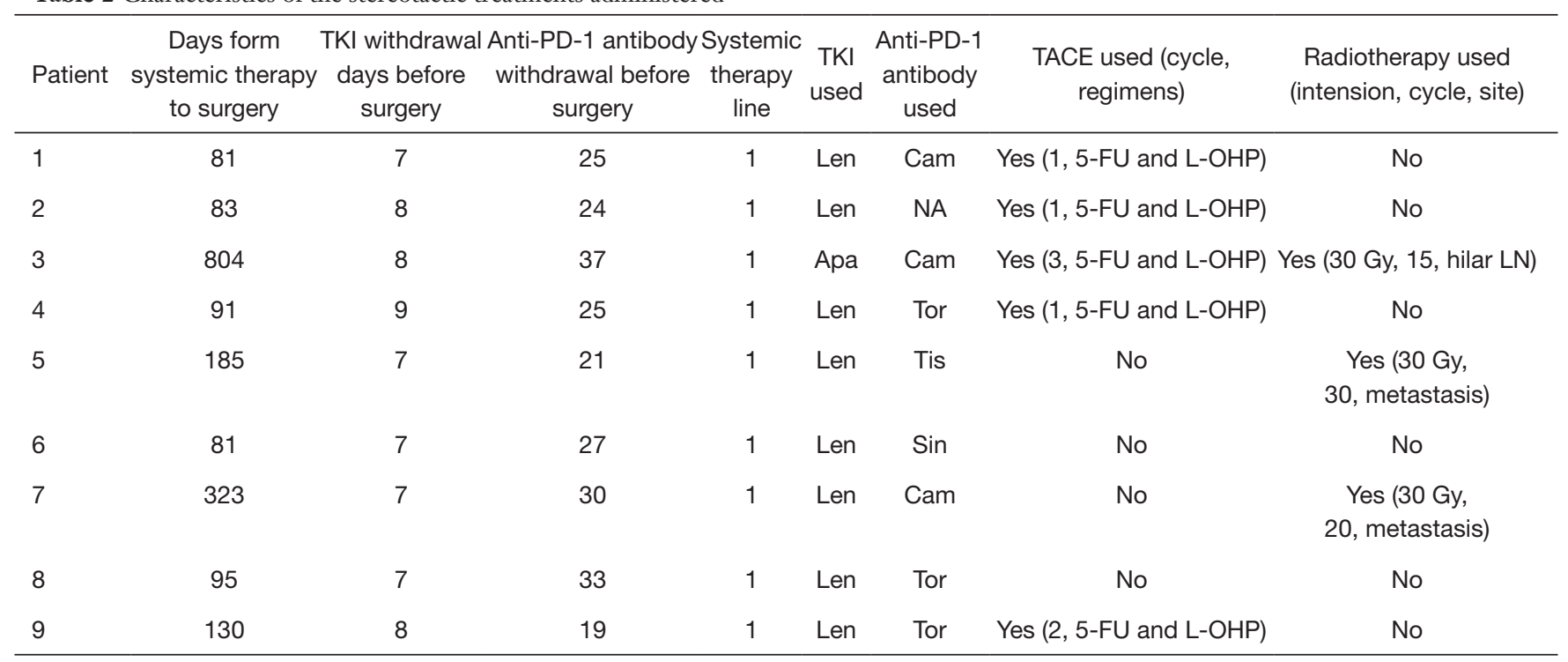

TKIs, tyrosine kinase inhibitors; PD-1, programmed death 1; Len, lenvatinib; Apa, apatinib; Sin, sintilimab; Cam, camrelizumab; Tis, tislelizumab; Tor, toripalimab; 5-FU, 5-fluorouracil; L-OHP, oxaliplatin; TACE, transarterial chemotherapy and embolization

\section{Follow-up}

The cut-off date for the present analysis was March 31, 2021. The median follow-up time was 10.2 (IQR, 8.6-20.0) months. Tumour recurrence was detected in 2 patients who received locoregional therapy plus lenvatinib and anti-PD-1 antibodies, but they were tumour-free at the last follow-up; the other patients were tumour-free. All 9 patients received stereotactic therapy and regular surveillance.

\section{Discussion}

Compared with the published literature, this is the first study to analyse therapeutic outcomes in patients receiving stereotactic therapy for CNLC stage IIIB HCC, and the results of this study show that stereotactic therapy can successfully convert these patients to resectable status. The results indicate that the combination of TKIs, ICIs and local regional therapy is a feasible conversion therapy for patients with CNLC stage IIIB HCC to achieve successful resection and has the potential to improve long-term survival.

Previous studies have indicated that the objective response rate of single use of TKIs or ICIs is approximately $15 \%(7,17)$. Due to delays in updates to the guidelines, many advanced HCC patients have a poor chance of receiving alternative treatments after monotherapy failure, and while these patients seem healthy, they are resigned to wait for death. Fortunately, several studies in 2020 revealed that immune drugs combined with targeted drugs can improve the objective response rate of advanced hepatocellular carcinoma. In patients with unresectable advanced hepatocellular carcinoma, the highest objective response rate can be achieved with a combination of atezolizumab and bevacizumab, pembrolizumab and lenvatinib, and camrelizumab and apatinib, reaching up to approximately $45 \%(8,16,18)$. After much clinical practice and review, our centre found that, on the basis of TKIs and ICIs, adding local regional treatment at an early time points tends to extend survival (19). TACE and TKIs plus ICIs have a synergistic effect, so the combined effect is better. In addition, some clinical trials are investigating immunization combined with local treatments, such as pembrolizumab and lenvatinib and TACE. It has been reported that radiotherapy has an abscopal effect on some tumours and that immunotherapy can enhance this effect $(20,21)$. In our research, we found that after radiotherapy of abdominal lesions, the primary lesions would also shrink, which demonstrate the synergistic effect of local treatment, and it also illustrates the importance of local regional therapy. In real-world clinical practice, we found that an increasing number of patients can obtain a potential curative effect through TKIs plus ICIs and locoregional treatment, and these patients have a longer survival than that in previous study (22-24). 
A
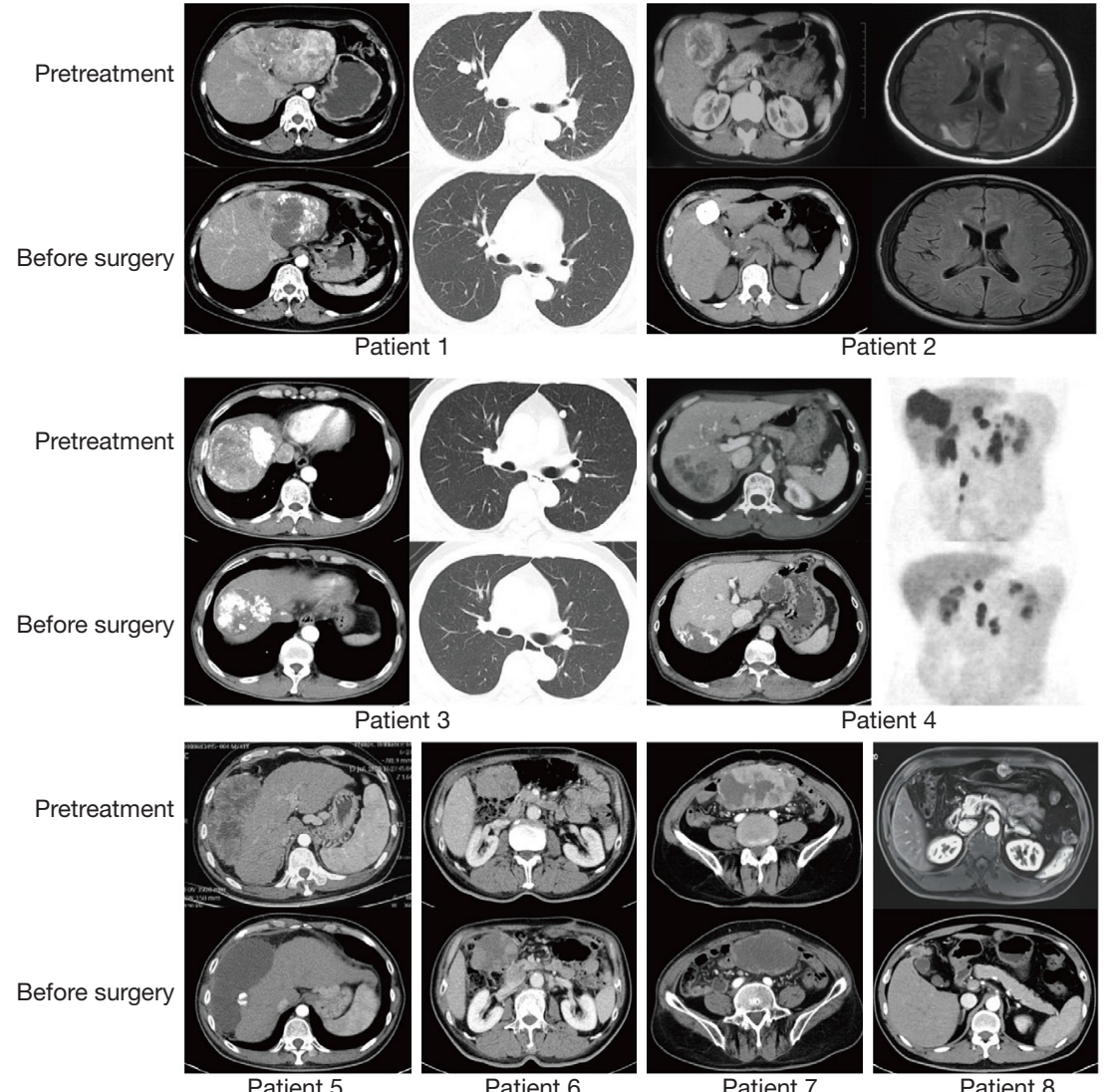

Patient 4

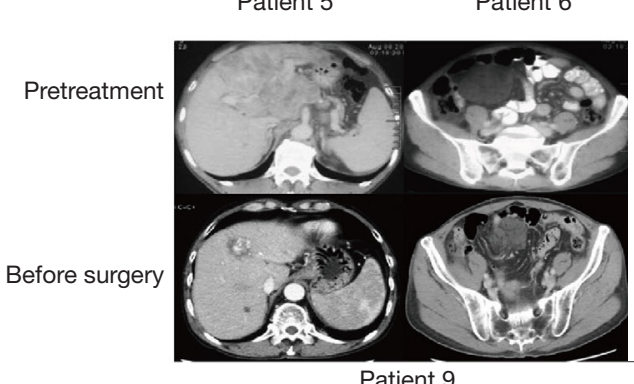

Patient 9

B

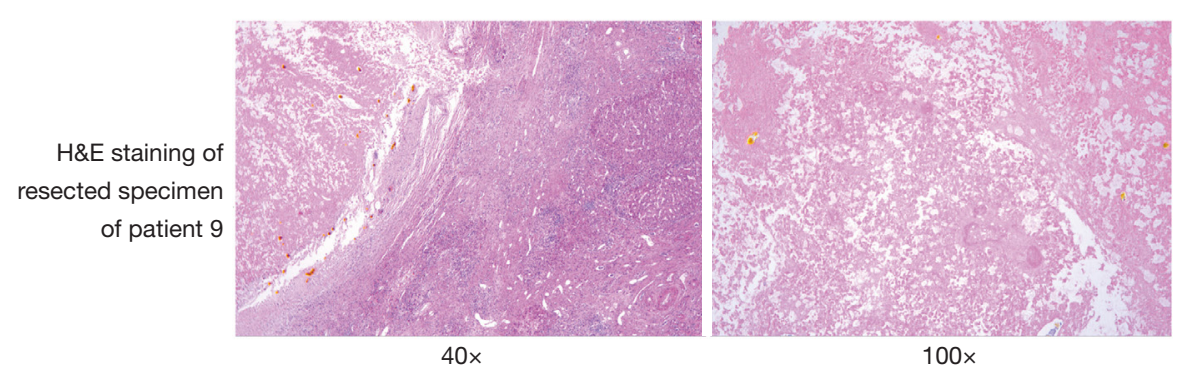

Figure 3 Pre-treatment and preoperative CT or MR scans and H\&E of resected regimen. (A) Pre-treatment MR or CT scans revealed that the patients had extrahepatic metastasis lesions. After stereotactic treatment, these metastatic lesions disappeared. (B) A representative case. Patient 9 was diagnosed with a solitary HCC and with an oligometastasis in lower abdominal cavity (BCLC stage C) and received TACE, radiotherapy, lenvatinib $8 \mathrm{mg}$ /day and toripalimab $240 \mathrm{mg}$ every 3 weeks. Four weeks later, the conventional operation was performed. H\&E staining of the surgically resected specimen showed a pCR. HCC, hepatocellular carcinoma; BCLC, Barcelona Clinic Liver Cancer; TACE, transarterial chemoembolization; H\&E, hematoxylin and eosin; $\mathrm{PCR}$, pathological complete response. 


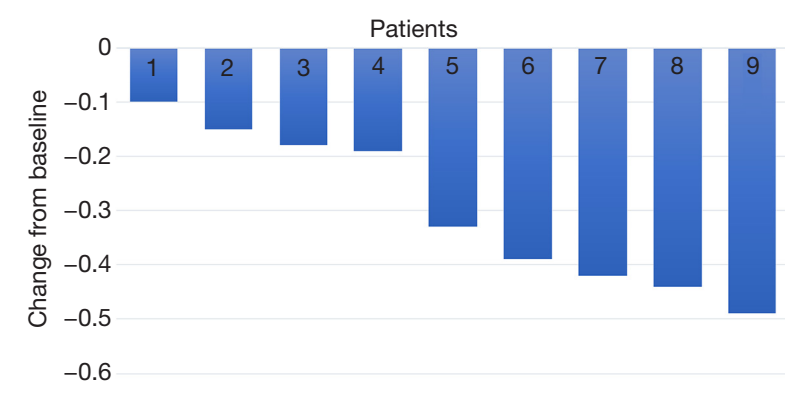

Figure 4 Maximum percent change in the target lesion from baseline. Therapeutic efficacy in patients with advanced hepatocellular carcinoma. Maximum percent change in the sum of the diameters of the target lesions from baseline.

One of the most encouraging aspects of the results is that the pCR rate was 33\% (3/9), which is slightly higher than previous research. These findings support the feasibility and efficacy of stereotactic therapy for CNLC stage IIIB HCC. We believe that the tumour must be completely removed, as relapse may occur otherwise. Another reason for the need for resection is that we do not know whether the patient achieved pCR before surgery, and there may be discrepancies between preoperative and intraoperative imaging. Another inspiring finding was that no MVI was found in all the 9 patients, suggesting that the surgical method should probably change. In order to ensure the surgical margin, the usage of traditional surgical method may lose a lot of liver volume, which may increase the risk of postoperative liver failure. However, in the era of stereotactic therapy, this old concept may be changed, and tumour removal should be the main goal.

No grade 4 or 5 treatment-related adverse events occurred during the stereotactic treatment period, so this treatment is relatively safe. The present study demonstrated that major hepatectomy is also safe during the perioperative period, and the surgical trauma and incidence of postoperative complications were similar to those in previous reports (25). The mechanism of TKIs may increase the risk of bleeding during hepatectomy and could lead to the delayed healing of wounds and incisions. ICIs may lead to immune-related inflammation in the liver and then to an increase in liver texture fragility. Intervention and radiotherapy may cause severe tissue adhesion and increase the difficulty of operation. Thus, after stereotactic therapy, the general condition of these patients was weak. In addition to preoperative drug withdrawal, great care should
Table 3 Surgical setting and pathological conditions

\begin{tabular}{lc}
\hline Parameters & Total $(\mathrm{N}=9)$ \\
\hline Surgical type, $\mathrm{n}(\%)$ & $3(33.3)$ \\
Open & $6(66.7)$ \\
Laparoscopic & $200(190$ to 220$)$ \\
Time of surgery, mins (median, IQR) & $200(100$ to 220$)$ \\
Bleeding, mL (median, IQR) & $0(0$ to 0$)$ \\
RBC transfusion, mL (median, IQR) & $0(0$ to 18) \\
Time of PV block, mins (median, IQR) & $6(5$ to 8$)$ \\
Postoperative hospital day, days (median, & \\
IQR) & \\
Differentiated histology, $\mathrm{n}(\%)$ & $3(33.3)$ \\
Moderate & $2(22.2)$ \\
Moderate-poor & $1(11.1)$ \\
Moderate-high & $3(33.3)$ \\
pCR & $0(0.0)$ \\
MVI, n (\%)
\end{tabular}

RBC, red blood cell; PV, portal vein; MVI, microvascular invasion.

Table 4 Safety summary

\begin{tabular}{lcc}
\hline AEs & Any grade, $\mathrm{n}(\%)$ & Grade 3, $\mathrm{n}(\%)$ \\
\hline Fatigue & $6(66.7)$ & $1(11.1)$ \\
Hypertension & $5(55.6)$ & $2(22.2)$ \\
ALT or AST elevation & $6(66.7)$ & $0(0.0)$ \\
Hypothyroidism & $1(11.1)$ & $0(0.0)$ \\
Decreased appetite & $5(55.6)$ & $1(11.1)$ \\
Abdominal pain & $1(11.1)$ & $0(0.0)$ \\
Vomiting & $2(22.2)$ & $0(0.0)$ \\
Skin rash & $3(33.3)$ & $1(11.1)$ \\
Anaemia & $1(11.1)$ & $0(0.0)$ \\
Diarrhoea & $1(11.1)$ & $0(0.0)$ \\
Hand-foot syndrome & $2(22.2)$ & $0(0.0)$ \\
Leukocytopenia & $2(22.2)$ & $1(11.1)$ \\
Nasal haemorrhage & $4(44.4)$ & $1(11.1)$ \\
Asthma & $1(11.1)$ & $1(11.1)$ \\
\hline AEs, adverse events; ALT, & &
\end{tabular}

AEs, adverse events; ALT, alanine aminotransferase; AST, aspartate aminotransferase. 
be taken when performing surgery. As conditions permit, laparoscopic surgery should be used, and surgical trauma should be minimized.

The limitations of our exploratory analysis must be acknowledged. First, the sample size of this study is small, and this study describes real-world cases rather than following a regular prospective RCT design. Therefore, the level of evidence is relatively low. Second, the followup time after surgery was relatively short, so the long-term efficacy is unknown. Third, the identification of patients who could be successfully converted to surgery was not exact; more research is needed in this area. Finally, the TKIs, ICIs and locoregional treatment used in this study were not unified. In real-world practice, the choice of suitable patients will be an important consideration.

\section{Conclusions}

In conclusion, we reported the outcomes of 9 patients with initially unresectable CNLC stage IIIB HCC who were successfully converted to surgery after stereotactic therapy. These patients tend to obtain a longer period of tumourfree survival, which may provide evidence for clinical practice. And the early addition of locoregional therapy to systemic therapy may confer better efficacy, downstaging and conversion to surgery, thereby leading to long-term survival. Meanwhile, patients with lung metastases and/or lymph node metastases seem to be more likely to converted to surgery and get clinical benefit. Although the outcomes of the patients presented herein seem encouraging, the exact role of stereotactic therapy and subsequent liver resection in this patient population requires further confirmation in prospective randomized controlled trials.

\section{Acknowledgments}

We thank the patients participating in this study and all staff at the hospital for their contributions to this study.

Funding: This work was supported by International Science and Technology Cooperation Projects (2016YFE0107100), CAMS Clinical and Translational Medicine Research Funds (2019XK320006), CAMS Innovation Fund for Medical Science (CIFMS) (2017-I2M-4-003 and 2018I2M-3-001), Beijing Natural Science Foundation (L172055 and 7192158), the Fundamental Research Funds for the Central Universities (3332018032), CSCO-Hengrui Cancer Research Fund (Y-HR2019-0239) and National Tenthousand Talent Program.

\section{Footnote}

Reporting Checklist: The authors have completed the STROBE reporting checklist. Available at https://hbsn. amegroups.com/article/view/10.21037/hbsn-21-188/rc

Data Sharing Statement: Available at https://hbsn. amegroups.com/article/view/10.21037/hbsn-21-188/dss

Conflicts of Interest: All authors have completed the ICMJE uniform disclosure form (available at https://hbsn. amegroups.com/article/view/10.21037/hbsn-21-188/coif). Drs. XL, YM, HZ and XS serve as the unpaid editorial board members of Hepatobiliary Surgery and Nutrition. The other authors have no conflicts of interest to declare.

Ethical Statement: The authors are accountable for all aspects of the work in ensuring that questions related to the accuracy or integrity of any part of the work are appropriately investigated and resolved. The study was conducted in accordance with the Declaration of Helsinki (as revised in 2013). And the study was approved by the Institutional Review Board (IRB) and Ethics Committee (EC) of Peking Union Medical College Hospital (PUMCH) (approval number: JS-1391) and informed consent was taken from all individual participants.

Open Access Statement: This is an Open Access article distributed in accordance with the Creative Commons Attribution-NonCommercial-NoDerivs 4.0 International License (CC BY-NC-ND 4.0), which permits the noncommercial replication and distribution of the article with the strict proviso that no changes or edits are made and the original work is properly cited (including links to both the formal publication through the relevant DOI and the license). See: https://creativecommons.org/licenses/by-nc-nd/4.0/.

\section{References}

1. Bray F, Ferlay J, Soerjomataram I, et al. Global cancer statistics 2018: GLOBOCAN estimates of incidence and mortality worldwide for 36 cancers in 185 countries. CA Cancer J Clin 2018;68:394-424.

2. Zhang S, Sun K, Zheng R, et al. Cancer incidence and mortality in China, 2015. J Nat Cancer Cent 2021;1:2-11.

3. Vogel A, Cervantes A, Chau I, et al. Hepatocellular carcinoma: ESMO Clinical Practice Guidelines for diagnosis, treatment and follow-up. Ann Oncol 
2018;29:iv238-55.

4. European Association For The Study Of The Liver; European Organisation For Research And Treatment Of Cancer. EASL-EORTC clinical practice guidelines: management of hepatocellular carcinoma. J Hepatol 2012;56:908-43.

5. Llovet JM, Ricci S, Mazzaferro V, et al. Sorafenib in advanced hepatocellular carcinoma. N Engl J Med 2008;359:378-90.

6. Cheng AL, Kang YK, Chen Z, et al. Efficacy and safety of sorafenib in patients in the Asia-Pacific region with advanced hepatocellular carcinoma: a phase III randomised, double-blind, placebo-controlled trial. Lancet Oncol 2009;10:25-34.

7. Kudo M, Finn RS, Qin S, et al. Lenvatinib versus sorafenib in first-line treatment of patients with unresectable hepatocellular carcinoma: a randomised phase 3 noninferiority trial. Lancet 2018;391:1163-73.

8. Finn RS, Qin S, Ikeda M, et al. Atezolizumab plus Bevacizumab in Unresectable Hepatocellular Carcinoma. N Engl J Med 2020;382:1894-905.

9. Zhang T, Merle P, Wang H, et al. Combination therapy for advanced hepatocellular carcinoma: do we see the light at the end of the tunnel? Hepatobiliary Surg Nutr 2021;10:180-92.

10. Sato N, Beppu T, Kinoshita K, et al. Conversion Hepatectomy for Huge Hepatocellular Carcinoma With Arterioportal Shunt After Chemoembolization and Lenvatinib Therapy. Anticancer Res 2019;39:5695-701.

11. Takeda K, Tsurumaru Y, Yamamoto Y, et al. Treatment of hepatocellular carcinoma with hepatic vein tumor thrombosis protruding into the inferior vena cava by conversion surgery following chemotherapy with regorafenib: a case report. Clin J Gastroenterol 2020;13:428-33.

12. Schwacha-Eipper B, Minciuna I, Banz V, et al. Immunotherapy as a Downstaging Therapy for Liver Transplantation. Hepatology 2020;72:1488-90.

13. Marrero JA, Kulik LM, Sirlin CB, et al. Diagnosis, Staging, and Management of Hepatocellular Carcinoma: 2018 Practice Guidance by the American Association for the Study of Liver Diseases. Hepatology 2018;68:723-50.

14. Lencioni R, Llovet JM. Modified RECIST (mRECIST) assessment for hepatocellular carcinoma. Semin Liver Dis 2010;30:52-60.

15. Pitroda SP, Chmura SJ, Weichselbaum RR. Integration of radiotherapy and immunotherapy for treatment of oligometastases. Lancet Oncol 2019;20:e434-42.

16. Zhu AX, Finn RS, Edeline J, et al. Pembrolizumab in patients with advanced hepatocellular carcinoma previously treated with sorafenib (KEYNOTE-224): a non-randomised, open-label phase 2 trial. Lancet Oncol 2018;19:940-52.

17. Finn RS, Ikeda M, Zhu AX, et al. Phase Ib Study of Lenvatinib Plus Pembrolizumab in Patients With Unresectable Hepatocellular Carcinoma. J Clin Oncol 2020;38:2960-70.

18. Xu J, Shen J, Gu S, et al. Camrelizumab in Combination with Apatinib in Patients with Advanced Hepatocellular Carcinoma (RESCUE): A Nonrandomized, Open-label, Phase II Trial. Clin Cancer Res 2021;27:1003-11.

19. Lin J, Zhao H. Systemic management for patients with hepatobiliary tumors in a multi-dimensional view. Hepatobiliary Surg Nutr 2019;8:626-8.

20. Komatsu T, Nakamura K, Kawase A. Abscopal Effect of Nivolumab in a Patient with Primary Lung Cancer. J Thorac Oncol 2017;12:e143-4.

21. Postow MA, Callahan MK, Barker CA, et al. Immunologic correlates of the abscopal effect in a patient with melanoma. N Engl J Med 2012;366:925-31.

22. Natsuizaka M, Omura T, Akaike T, et al. Clinical features of hepatocellular carcinoma with extrahepatic metastases. J Gastroenterol Hepatol 2005;20:1781-7.

23. Lee IC, Huo TI, Huang YH, et al. Transarterial chemoembolization can prolong survival for patients with metastatic hepatocellular carcinoma: a propensity score matching analysis. Hepatol Int 2012;6:753-62.

24. Yoo DJ, Kim KM, Jin YJ, et al. Clinical outcome of 251 patients with extrahepatic metastasis at initial diagnosis of hepatocellular carcinoma: does transarterial chemoembolization improve survival in these patients? J Gastroenterol Hepatol 2011;26:145-54.

25. Chen ZL, Zhang CW, Liang L, et al. Major Hepatectomy in Elderly Patients with Large Hepatocellular Carcinoma: A Multicenter Retrospective Observational Study. Cancer Manag Res 2020;12:5607-18.

Cite this article as: Yang $\mathrm{X}, \mathrm{Xu} \mathrm{H}$, Zuo B, Yang $\mathrm{X}$, Bian $\mathrm{J}$, Long J, Wang D, Zhang J, Ning C, Wang Y, Xun Z, Wang Y, Lu X, Mao Y, Sang X, Zhao H. Downstaging and resection of hepatocellular carcinoma in patients with extrahepatic metastases after stereotactic therapy. HepatoBiliary Surg Nutr 2021;10(4):434-442. doi: 10.21037/hbsn-21-188 